\title{
Avaliação da força muscular respiratória em crianças e adolescentes com sobrepeso/obesos
}

\author{
Evaluation of respiratory muscle strength in overweight/obese children and adolescents
}

\author{
Sandra Quintino Santiago ${ }^{1}$, Maria de Lourdes P. da Silva², Josy Davidson ${ }^{3}$, Luciana Ritha de C. R. B. Aristóteles ${ }^{4}$
}

\section{RESUMO}

Objetivo: Avaliar a força muscular respiratória de crianças e adolescentes com sobrepeso ou obesidade.

Métodos: Estudo transversal com crianças e adolescentes entre quatro e 15 anos de idade de duas instituições de ensino fundamental e uma clínica de nutrição. As crianças foram avaliadas e classificadas em dois grupos, de acordo com a curva proposta pelo National Center for Health Statistics: sobrepeso/ obesos (GSO, índice de massa corpórea (IMC) em relação à idade e ao sexo acima do percentil 85) e eutróficos (GE, IMC entre percentil 5 e 85). Para avaliar as pressões inspiratória máxima (PImax) e expiratória máxima (PEmax) foram realizadas três medidas com um manovacuômetro, considerando-se a maior medida a partir da capacidade máxima inspiratória e expiratória. Aplicou-se o teste $t$ para as variáveis quantitativas e o qui-quadrado para as qualitativas. Para ajuste das covariáveis, foi feita a análise de covariância, sendo significante $p<0,05$.

Resultados: Foram avaliadas 69 crianças: 37 (54\%) do GSO e 32 (46\%) eutróficos. O GSO apresentou menor idade $(9,8 \pm 2,3$ versus $10,9 \pm 1,9$ anos; $p=0,03)$. A PImax foi $71,4 \pm 24,9 \mathrm{cmH}_{2} \mathrm{O}$ no GSO e $89,6 \pm 19,6 \mathrm{cmH}_{2} \mathrm{O}$ nos eutróficos $(p=0,002)$. A PEmax foi $71,9 \pm 24,8 \mathrm{cmH}_{2} \mathrm{O}$ no GSO e 95,6 $\pm 19,6 \mathrm{cmH}_{2} \mathrm{O}$ nos eutróficos $(p<0,001)$. Não houve diferenças quanto ao sexo e à prática de atividade física. Ajustando-se os valores em relação à idade, somente a PEmax manteve-se diferente entre os grupos $(p=0,003)$.

Conclusões: A força muscular expiratória mostrou-se diminuída nesta amostra de crianças e adolescentes com sobrepeso/obesidade, indicando que a obesidade pode comprometer a mecânica pulmonar dessa população.

Palavras-chave: sobrepeso; obesidade; força muscular; pulmão; criança.

'Especialista em Fisioterapia Respiratória pela Universidade Federal de São Paulo (Unifesp), São Paulo, SP, Brasil

Especialista em Fisioterapia em Clínica Médica pela Unifesp, São Paulo, $\mathrm{SP}$, Brasil

${ }^{3}$ Mestre em Ciências pela Unifesp e supervisora do curso de especialização em Fisioterapia Respiratória da Unifesp, São Paulo, SP, Brasil

${ }^{4}$ Especialista em Fisioterapia Cardiorrespiratória pela Faculdade de Ciências Médicas de Minas Gerais, supervisora de Unidade de Terapia Intensiva Adulto do curso de Fisioterapia da Universidade Guarulhos, fisioterapeuta do Hospital Alemão Oswaldo Cruz, São Paulo, SP, Brasil

\section{ABSTRACT}

Objective: Evaluate respiratory muscle strength in overweight/obese children and adolescents.

Methods: This cross-sectional study enrolled patients between four and 15 years old, classified according to the National Center for Health Statistics curve for body mass index (BMI): Overweight/Obese Group (OG, BMI> 85 ${ }^{\text {th }}$ percentile) and Normal Weight Group (NG, BMI between $5^{\text {th }}$ and $85^{\text {th }}$ percentile). Manuvacuometer was used to measure maximal inspiratory pressure (MaxInspP) and maximal expiratory pressure (MaxExpP). Three measurements were obtained using the maximum pulmonary effort and the higher value was considered. Variables were compared by $t$ test chi-square test. Adjustment of possible covariants was made by analysis of covariance, being significant $p<0.05$.

Results: 69 children were included: $37(54 \%)$ in the OG and $32(46 \%)$ in the NG. Obese patients were younger: $9.8 \pm 2.3$ versus $10.9 \pm 1.9$ years $(p=0.031)$. MaxInspP was $71.4 \pm 24.9 \mathrm{cmH}_{2} \mathrm{O}$ in the $\mathrm{OG}$ and $89.6 \pm 19.6 \mathrm{cmH}_{2} \mathrm{O}$ $(p=0.002)$. MaxExpP was $71.9 \pm 24.8 \mathrm{cmH}_{2} \mathrm{O}$ in the OG and $95.6 \pm 19.6 \mathrm{cmH}_{2} \mathrm{O}$ in the $\mathrm{NG}(p<0.001)$. There was no difference between groups regarding gender and physical activity. After adjustment for age, only the MaxExpP was significantly different between groups ( $p=0.003)$.

Conclusions: Maximal expiratory pressure was lower in overweight/obese patients, indicating that obesity may alter pulmonary mechanics.

Key-words: overweight; obesity; muscle strength; lung; child.

Endereço para correspondência:

Sandra Quintino Santiago

Rua Mucuri, 70, apto. 61, Vila Guarani

CEP 04313-050 - São Paulo/SP

E-mail: sandrasantiago@terra.com.br

Recebido em: 6/9/2007

Aprovado em: 18/3/2008 


\section{Introdução}

A obesidade tem se tornado um problema de Saúde Pública em todo o mundo. Nos últimos dez anos, a prevalência da obesidade aumentou em torno de 10 a $40 \%$ na maioria dos paises europeus ${ }^{(1)}$. No Brasil, segundo a Pesquisa de Orçamentos Familiares de 2002-2003 ${ }^{(2)}$, 40\% da população adulta apresenta excesso de peso, sendo que cerca de $20 \%$ dos homens são classificados como obesos e um terço das mulheres estão com excesso de peso.

O número de crianças e adolescentes obesos também tem crescido de forma significativa. No Brasil, houve um aumento na prevalência da obesidade de 4,1\% em 1974 para $14 \%$ em 2002-2003, na população entre seis e 18 anos $^{(3)}$. Tal aumento foi detectado até mesmo em regiões que, historicamente, apresentam sérios problemas de desnutrição, como o Norte e o Nordeste ${ }^{(4)}$.

O aumento no número de obesos na infância e adolescência contribui de forma direta para a manutenção da obesidade na fase adulta, com conseqüente elevação de doenças associadas à mortalidade, como as cardiovasculares, a síndrome metabólica, a hiperlipidemia, a diabetes, a hipertensão e o acidente vascular encefálico ${ }^{(5-7)}$.

Além dos problemas acima descritos, a obesidade pode trazer ainda alterações na mecânica respiratória, causadas pelo acúmulo de gordura nas costelas, diafragma e abdome, reduzindo a complacência da caixa torácica e diminuindo a excursão diafragmática, o que pode levar a um maior consumo de oxigênio para a respiração(8-10).

Apesar de a relação entre sobrepeso/obesidade e pressões respiratórias ser descrita em alguns estudos, tal associação não está bem esclarecida em crianças e adolescentes. Portanto, o objetivo deste estudo foi avaliar a força muscular respiratória de crianças e adolescentes com sobrepeso/obesidade.

\section{Métodos}

Estudo transversal com crianças e adolescentes entre quatro e 15 anos, em três instituições, sendo duas de ensino fundamental (uma privada e uma pública) e uma clínica de nutrição, no período de agosto a setembro de 2005.

O estudo foi aprovado pelo Comitê de Ética da Universidade Guarulhos (UnG) e a coleta dos dados foi realizada após a assinatura do termo de consentimento livre e esclarecido pelo responsável. Para inclusão dos pacientes no estudo, um profissional de cada instituição foi orientado antecipadamente a respeito da pesquisa, sendo solicitado que fossem selecionadas crianças e adolescentes com o peso ideal para altura e outros acima do peso, de acordo com o parecer do profissional. Crianças com doenças respiratórias, cardíacas ou neuromusculares relatadas pelos pais, dificuldade de compreensão e/ou na execução das medidas foram excluídas do estudo.

Um questionário com dados pessoais, hábitos alimentares e prática de atividade física foi preenchido pelo pesquisador por meio de entrevista com o responsável pela criança. Foi considerada prática de atividade física qualquer atividade realizada por, no mínimo, 40 minutos, pelo menos três vezes na semana, executadas na escola ou não. Além disso, o responsável era questionado sobre seu parecer em relação à condição física da criança, considerando-a obesa ou não.

Os dados antropométricos foram verificados com uma balança digital Marte ${ }^{\circledR}$, modelo PP180 (SP-Brasil), com capacidade de 0 a $150 \mathrm{~kg}$ e precisão de $100 \mathrm{~g}$. Para essa mensuração, o paciente permanecia descalço, com roupas leves, cabeça em linha média, braços ao longo do corpo e pés paralelos. Para mensurar a estatura, foi utilizado um estadiômetro SECA bodymeter $^{\circledR} 208$ (SP-Brasil), com precisão em centímetros e metros, variando de 0 a $2 \mathrm{~m}$ do chão. A criança/adolescente era posicionada pelo examinador para que a mensuração fosse fidedigna, com os pés paralelos, braços ao longo do corpo, cabeça em linha média, tomando o cuidado para que o quadril ficasse em posição neutra e a coluna torácica, encostada na parede. O cálculo do índice de massa corpórea (IMC) foi obtido por meio da divisão do peso corporal $(\mathrm{kg})$ pela estatura (m) elevada ao quadrado $\left(E^{2}\right)$.

A classificação dos pacientes como eutróficos (GE) ou com sobrepeso/obesidade (GSO) se baseou na curva do IMC em relação à idade e ao sexo de crianças/adolescentes entre dois e 20 anos de idade proposta pelo National Center for Health Statistics (NCHS), no ano 2000 ${ }^{(11,12)}$. Considerou-se sobrepeso/ obeso quando o IMC estava acima do percentil 85 .

A avaliação respiratória foi realizada com um manovacuômetro MPG $^{\circledast}$ (Marshalltown, EUA) com graduação de 0 a $120 \mathrm{cmH}_{2} \mathrm{O}$ e variações a cada $4 \mathrm{cmH}_{2} \mathrm{O}$, tanto para pressão inspiratória (PI) quanto para pressão expiratória (PE), com o objetivo de verificar a força muscular inspiratória e expiratória.

A pressão expiratória máxima (PEmax) foi obtida com a criança/adolescente sentada com um clip nasal e orientada a realizar a máxima inspiração, ou seja, a mensuração começava a partir da capacidade pulmonar total (CPT), sendo realizado um esforço máximo expiratório contra a válvula ocluída. Já para a pressão inspiratória máxima (PImax), o paciente iniciava o teste com a máxima expiração, ou seja, até o vo- 
lume residual, e aí realizava o esforço máximo inspiratório contra a válvula ocluída ${ }^{(13)}$. Para que as crianças/adolescentes executassem corretamente o teste, este foi demonstrado previamente por um adulto, que empregou sentenças de fácil compreensão. Assim, para avaliar a PEmax foi utilizada a frase "Assopre uma vela bem forte e o mais rápido possivel" e, para a PImax, a frase utilizada foi "Chupe o canudinho bem forte". A avaliação era iniciada somente após a devida compreensão da criança. O paciente permanecia sentado, com os pés apoiados ao chão, joelhos em flexão de $90^{\circ}$, quadril encaixado e coluna apoiada no encosto da cadeira, cabeça em linha média e mãos segurando o bucal. O pesquisador permaneceu à frente do paciente, segurando o manovacuômetro.

Tabela 1 - Características da amostra dos Grupos Sobrepeso/ Obesos (GSO) e Eutróficos (GE)

\begin{tabular}{lccc}
\hline & $\begin{array}{c}\text { GE } \\
\mathbf{n = 3 2}\end{array}$ & $\begin{array}{c}\text { GSO } \\
\mathbf{n = 3 7}\end{array}$ & $\boldsymbol{p}$ \\
\hline Idade (anos) & $10,9 \pm 1,9$ & $9,8 \pm 2,3$ & 0,031 \\
Peso $(\mathrm{kg})$ & $37,0 \pm 6,2$ & $52,2 \pm 18,0$ & 0,001 \\
Altura $(\mathrm{cm})$ & $144,6 \pm 10,8$ & $142,2 \pm 13,4$ & 0,401 \\
IMC $\left(\mathrm{kg} / \mathrm{m}^{2}\right)$ & $17,5 \pm 1,6$ & $24,8 \pm 4,8$ & $<0,001$ \\
Sexo feminino & $18(56 \%)$ & $24(65 \%)$ & 0,468 \\
Prática de AF & $22(69 \%)$ & $18(49 \%)$ & 0,094 \\
\hline
\end{tabular}

AF: atividade física.

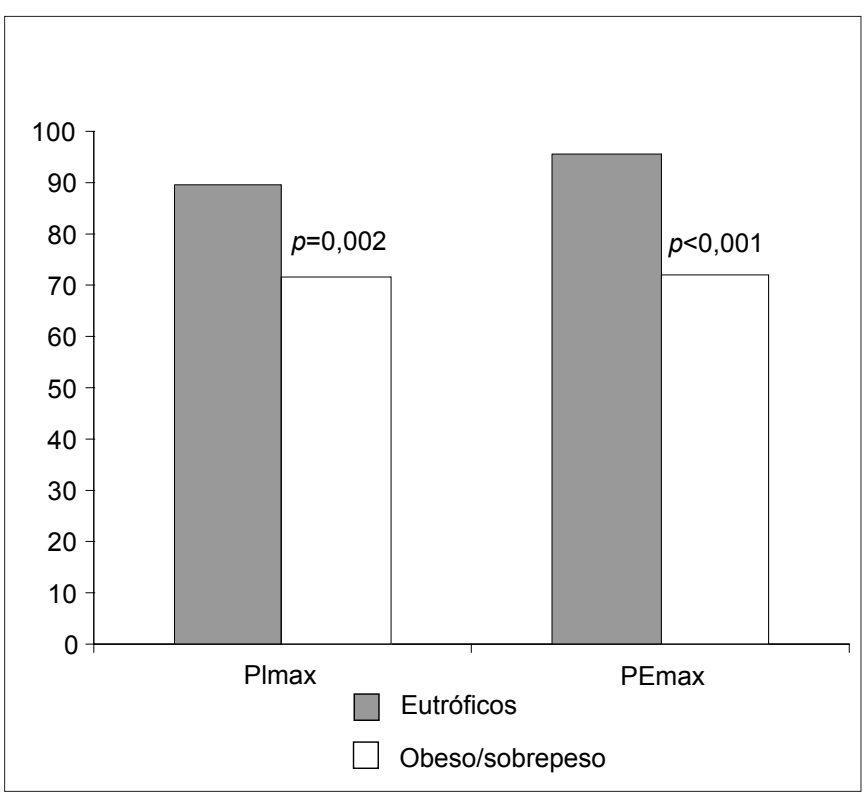

Gráfico 1 - Valores médios da PImax e PEmax nos Grupos Eutróficos e Sobrepeso/Obesos.
Para maior reprodutibilidade da mensuração, as medidas de PImax e de PEmax foram realizadas três vezes, sendo considerada a de maior valor, desde que este não ultrapassasse $20 \%$ das demais medidas. Caso houvesse discrepância entre as medidas, uma nova medida era realizada.

Todas as variáveis contínuas foram expressas em média e desvio padrão, visto que apresentavam distribuição normal. Para as variáveis categóricas, a comparação entre os grupos foi feita pelo teste do qui-quadrado e, para as numéricas, aplicouse o teste $t$ de Student não pareado. A análise de covariância (ANCOVA) $)^{(14,15)}$ foi empregada para ajustar as covariáveis: idade, sexo e prática de atividade física. Considerou-se significante $p<0,05$. Toda a análise foi feita com o Statistical Package for the Social Sciences for Windows, versão 12.0.1.

\section{Resultados}

Foram avaliados 69 pacientes: $37(54 \%)$ no GSO e $32(46 \%)$ no GE. As características da população estudada estão apresentadas na Tabela 1. Não houve diferenças entre os grupos em relação ao gênero, prática de atividade física e altura. As crianças do GSO eram mais jovens do que as do GE $(p=0,03)$. Além disso, apenas 22\% (8/37) dos pacientes do GSO foram classificados como sobrepeso, ou seja, com IMC entre os percentis 85 e 95 .

$\mathrm{Na}$ análise univariada, os valores de PImax e PEmax nos eutróficos foram maiores do que nos pacientes com sobrepeso/obesidade (Gráfico 1). As PImax foram respectivamente $71,4 \pm 24,9 \mathrm{cmH}_{2} \mathrm{O}$ no $\mathrm{GSO}$ e $89,6 \pm 19,6 \mathrm{cmH}_{2} \mathrm{O}$ no $\mathrm{GE}$ $(p=0,002)$. Já as PEmax foram $71,9 \pm 24,8 \mathrm{cmH}_{2} \mathrm{O}$ no GSO e $95,6 \pm 19,6 \mathrm{cmH}_{2} \mathrm{O}$ no $\mathrm{GE}(p<0,001)$. O poder do teste para a comparação da PImax foi $80 \%$ e para a PEmax foi $78 \%$.

No entanto, devido à diferença dos grupos em relação à idade e, na tentativa de eliminar a influência das covariáveis nos resultados, foi realizada a ANCOVA. Essa análise demonstrou que a idade influenciou nos valores da PImax $(p=0,002)$, com tendência à permanência das diferenças entre o GSO e o GE quanto a esta variável ( $p=0,068)$. Já em relação à PEmax, a idade não influenciou a força muscular expiratória do GSO $(p=0,313)$ e manteve-se a diferença entre os grupos $(p=0,003)$. Outras covariáveis, como sexo $(p=0,995)$ e atividade física $(p=0,726)$, não demonstraram influência sobre a força muscular respiratória.

Quanto à percepção das mães em relação às condições físicas de seus filhos, observou-se que $43 \%$ (16/37) das mães de crianças com sobrepeso/obesidade não classificaram seus filhos como portadores de peso excessivo para a altura. 


\section{Discussão}

Diferenças na avaliação da força muscular respiratória em pacientes adultos obesos são descritas há décadas ${ }^{(1,8,9,16,17)}$; entretanto, tal relação não está bem estabelecida em crianças obesas. O fato de esse estudo ter encontrado diferenças apenas nos valores de PEmax, quando comparados os portadores de sobrepeso/obesidade com os eutróficos, demonstra que o excesso de peso durante o crescimento pode gerar restrição pulmonar devido à diminuição da excursão diafragmática, causada pelo aumento da adiposidade abdominal ou pelo peso da parede torácica ${ }^{(8,10,16)}$. Tal diminuição também pode estar associada à deposição de gordura nos músculos, principalmente nos abdominais, responsáveis pela força expiratória. Com isso, haveria alterações progressivas na função pulmonar que, associadas ao crescimento, desencadeariam modificações na composição das fibras musculares, tanto em sua qualidade quanto em sua capacidade oxidativa, reduzindo, dessa forma, a força muscular respiratória ${ }^{(8,9)}$.

$\mathrm{A}$ influência da idade ${ }^{(18-21)}$ em relação às pressões respiratórias, independentemente dos valores antropométricos, já foi descrita até em estudos com adultos ${ }^{(17)}$. Em crianças e adolescentes, os fatores de crescimento ${ }^{(22)}$, os hormônio ${ }^{(19)}$ e o gênero ${ }^{(23)}$ podem ter maior influência sobre a força muscular respiratória do que os valores antropométricos ${ }^{(24,25)}$.

Um estudo ${ }^{(23)}$ com 235 adultos e crianças mostrou boa correlação entre a PImax e o peso, enquanto a PEmax apresentou maior correlação com a idade, contrariamente aos resultados aqui apresentados. Essa contradição pode se dever ao tipo de paciente e ao delineamento da pesquisa. No presente estudo, a casuística foi formada predominantemente por crianças e pré-adolescentes, sabendo-se que a fase de desenvolvimento pré- ou pós-puberal pode modificar a influência da idade nos valores da pressão inspiratória ou expiratória ${ }^{(19)}$.

Outro fator capaz de influenciar a força muscular respiratória é o gênero: homens apresentam maiores pressões respiratórias, quando comparados a mulheres adultas ${ }^{(18,20,21)}$. Nesse estudo, houve diferenças no número de meninas entre os grupos, porém, sem significância à análise univariada. $\mathrm{Na}$ ANCOVA, o gênero não mostrou influência nos resultados do estudo.

O fato de não haver diferenças entre os grupos quanto à prática de atividade física, contrariamente a outros estudos $^{(19)}$, poderia ser explicado por ter sido considerada atividade física qualquer atividade por tempo mínimo de 40 minutos, por três vezes na semana no mínimo. Talvez, se investigada de forma mais específica, sem levar em conta as atividades no período escolar, e se a casuística fosse maior, poderia haver diferenças entre os grupos.

Um dado interessante relaciona-se à percepção dos pais e/ou responsáveis quanto à condição física de seus filhos. A observação de que mais da metade dos pais de filhos com sobrepeso ou obesos não os classifica como tal demonstra a dificuldade para o tratamento da obesidade infantil, uma vez que a reeducação alimentar e o incentivo a condições de vida saudáveis, fundamentais para o bom desempenho do tratamento, dependem muito da participação dos pais.

A seleção de apenas algumas crianças/adolescentes das instituições participantes, pré-selecionadas pelo profissional de acordo com as características já mencionadas, limitou a análise em separado de crianças e adolescentes. Além disso, crianças limítrofes na curva do IMC provavelmente não foram selecionadas, visto que apenas a avaliação empírica e visual do profissional pode ter dificultado sua classificação em eutrófico ou sobrepeso. Com isso, apenas $20 \%$ das crianças incluídas no GSO apresentavam sobrepeso, o que pode ser uma limitação do estudo.

Considerando as alterações que geralmente decorrem do sobrepeso/obesidade, principalmente as de natureza pulmonar, justifica-se a necessidade de avaliações periódicas da força muscular respiratória em crianças com distúrbios nutricionais, com o objetivo de monitorizar as condições mecânicas do aparelho respiratório ${ }^{(26)}$. O estudo indica uma tendência à possível redução da PImax das crianças e adolescentes com sobrepeso/obesidade e uma significativa redução na PEmax. 


\section{Referências bibliográficas}

1. Jubber AS. Respiratory complications of obesity. Int J Clin Pract 2004;58:573-80.

2. Instituto Brasileiro de Geografia e Estatística [homepage on the Internet]. Pesquisa de Orçamentos Familiares - POF 2002-2003 [cited 2008 Feb 5]. Available from: http://www.ibge.gov.br/ibgeteen/datas/saude/obesidade.html

3. Instituto Brasileiro de Geografia e Estatística [homepage on the Internet]. Medidas Antropométricas de Crianças e Adolescentes 2002/2003 [cited 2008 Feb 5]. POF - Available from: www.ibge.gov.br/home/presidencia/noticias/ noticia_impressao.php?id_noticia $=625$

4. Fisberg M, Oliveira CL. Obesidade na infância e adolescência - uma verdadeira epidemia. Arq Bras Endocrinol Metab 2003;47:107-8.

5. Ministério do Planejamento, Orçamento e Gestão do Instituto Brasileiro de Geografia e Estatística. Pesquisas de orçamentos familiares 2002-2003 [monograph on the Internet]. Rio de Janeiro: IBGE; 2006 [cited 2007 May 5]. Available from: http://www.ibge.gov.br/home/estatistica/populacao/condicaodevida/pof/2003medidas/pof2003medidas.pdf

6. Wang Y, Monteiro C, Popkin BM. Trends of obesity and underweight in older children and adolescents in the United States, Brazil, China, and Russia. Am J Clin Nutr 2002;75:971-7.

7. Mello ED, Luft VC, Meyer F. Obesidade infantil: como podemos ser eficazes? J Pediatr (Rio J) 2004;80:173-82.

8. Naimark A, Cherniack RM. Compliance of the respiratory system and its components in health and obesity. J Appl Physiol 1960;15:377-82.

9. Rasslan Z, Saad Junior R, Stirbulov R, Fabbri RMA, Lima CAC. Avaliação da função pulmonar na obesidade graus I e II. J Bras Pneumol 2004;30:508-14.

10. Collins LC, Hoberty PD, Walker JF, Fletcher EC, Peiris AN. The effect of body fat distribution on pulmonary functions tests. Chest 1995;107:1298-302.

11. Must A, Dallal GE, Dietz WH. Reference data for obesity: 85th and 95th percentiles of body mass index (wt/ht2) and triceps skinfold thickness. Am J Clin Nutr 1991;53:839-46.

12. National Center for Heath Statistics [homepage on the Internet]. Body mass index-for-age. Children and adolescents, 2 to 20 years. United States: Individual Growth Charts CDC Growth Charts [cited 2008 Feb 5]. Available from: http:// www.cdc.gov/nchs/about/major/nhanes/growthcharts/clinical_charts.htm
13. American Thoracic Society/European Respiratory Society. ATS/ERS Statement on respiratory muscle testing. Am J Resp Crit Care Med 2002;166:518-624.

14. Magalhães MN, Lima ACP. Noções de probabilidade e estatística. $2^{\mathrm{a}}$ ed. São Paulo: IME-USP; 2000

15. Neter J, Kutner MH, Nachtsheim CJ, Wasserman W. Applied linear statistical. $4^{\text {th }}$ ed. Chicago: Irwin; 1996.

16. Domingos-Benício NC, Gastaldi AC, Perecin JC, Avena K de M, Guimarães RC Sologuren $\mathrm{MJ}$ et al. Spirometric values of obese and non-obese subjects on orthostatic, sitting and supine positions. Rev Assoc Med Bras 2004;50:142-7.

17. Black LF, Hyatt RE. Maximal respiratory pressures: normal values and relationship to age and sex. Am Rev Respir Dis 1969;99:696-702.

18. Gaultier C, Zinman R. Maximal static pressures in healthy children. Respir Physiol 1983;51:45-61.

19. Matecki S, Prioux J, Jaber S, Hayot M, Prefaut C, Ramonatxo M. Respiratory pressures in boys from 11-17 years old: a semilongitudinal study. Pediatr Pulmonol 2003;35:368-74

20. Domènech-Clar R, López-Andreu JA, Compte-Torrero L, De Diego-Damiá A Macián-Gisbert V, Perpiñá-Tordera $\mathrm{M}$ et al. Maximal static respiratory pressures in children and adolescents. Pediatr Pulmonol 2003;35:126-32.

21. Tomalak W, Pogorzelski A, Prusak J. Normal values for maximal static inspiratory and expiratory pressures in healthy children. Pediatr Pulmonol 2002;34:42-6.

22. Wagener JS, Hibbert ME, Landau LI. Maximal respiratory pressures in children Am Rev Respir Dis 1984;129:873-5.

23. Wilson SH, Cooke NT, Edwards RH, Spiro SG. Predicted normal values for maximal respiratory pressures in caucasians adults and children. Thorax 1984;39:535-8.

24. Bruschi C, Cerveri I, Zoia MC, Fanfulla F, Fiorentini M, Casali L et al. Reference values of maximal respiratory mouth pressures: a population based study. Am Rev Respir Dis 1992;146:790-93.

25. Smyth RJ, Chapmann KR, Rebuck AS. Maximal inspiratory and expiratory pressures in adolescents. Normal values. Chest 1984;86:568-72.

26. Costa D, Sampaio LMM, Lorenzzo VAP, Jamami M, Damaso AR. Avaliação da força muscular respiratória e amplitudes torácicas e abdominais após a RFR em indivíduos obesos. Rev Latino-Am Enfermagem 2003;11:156-60. 